167 MSC-CONCENTRATED SUPERNATANT: A NOVEL
THERAPEUTICAL APPROACH IN INFLAMMATION-INDUCED
PRETERM BRAIN INJURY?

doi:10.1136/archdischild-2012-302724.0167

${ }^{1} \mathrm{~K}$ Drommelschmidt, 'S Prager, 'I Bendix, ${ }^{2} \mathrm{M}$ Keller, ${ }^{3} \mathrm{PA}$ Horn, ${ }^{3}$ AK Ludwig, ${ }^{3} \mathrm{~S}$ Radtke, ${ }^{3} \mathrm{~B}$ Giebel, 'U Felderhoff-Müser. 'Department of Pediatrics I, University Children's Hospital, Essen; ${ }^{2}$ Childrens Hospital Passau, Passau; ${ }^{3}$ nnstitute of Transfusion Medicine, University of Essen, Essen, Germany

Background and aims Preterm brain injury of premature born infants is a main cause of disability and represents an enormous individual, familial and social burden. Up to $50 \%$ of these children suffer from disabilities such as cerebral palsy and cognitive disorders. The aetiology of brain injury has been considered to be multifactorial. Factors such as hyperoxia or inflammation are important pathomechanism in the development of brain injury.

As infections and subsequent inflammation are almost unavoidable on NICUs, new anti-inflammatory approaches are needed. In differend experimental settings, mesenchymal stem cells (MSCs) show these anti-inflammatory abilities. Although intravenously administered MSCs get trapped in the lung, therapeutic effects in target tissue are detectable, thereby indicating paracrine mechanisms. Since MSC-supernatants contain molecules with immunosuppressive functions, such as the identified TSG-6, we wondered whether MSC supernatants contain additional, maybe synergistically acting factors. The objective of this study is to evaluate the effect of concentrated MSC-supernatant on brain damage caused by inflammation.

Methods Wistar rats were randomized in 4 groups (vehicle/vehicle, vehicle/concentrated MSC-supernatant, LPS/vehicle, LPS/concentrated supernatant). LPS $(0.25 \mathrm{mg} / \mathrm{kg})$ was administered at $\mathrm{p} 3$, concentrated MSC-supernatant at $\mathrm{p} 3$ and $\mathrm{p} 4$. At p5, animals were transcardially perfused, brains were removed and snap-frozen for molecularbiological analysis.

Results At p5, LPS-treated animals show a marked increase in apoptosis, whereas additional treatment with concentrated MSCsupernatant results in a decrease in neural apoptosis.

Conclusions Concentrated MSC-supernatant showed promising effects on inflammation-induced brain damage in an experimental model of encephalopathy of prematurity.

\section{SERUM VEG F LEVELS IN PRETERM INFANTS: EFFECTS OF GESTATION AND LASER OR BEVACIZUMAB THERAPY FOR RETINOPATHY OF PREMATURITY}

doi:10.1136/archdischild-2012-302724.0168

1,2J Berrington, ${ }^{3} \mathrm{~A}$ Shafiq, ${ }^{4} \mathrm{I}$ Dimmick, ${ }^{1,2} \mathrm{ND}$ Embleton. 'Newcastle Neonatal Service, Newcastle upon Tyne Hospitals NHS Trust; ${ }^{2}$ Institute of Health and Society, Newcastle University; ${ }^{3}$ Ophthalmology, Newcastle upon Tyne Hospitals NHS Trust; ${ }^{4}$ Faculty of Medical Sciences, Newcastle University, Newcastle upon Tyne, UK

Background Bevacizumab, a monoclonal antibody to the vascular endothelial growth factor Veg F appears to offer successful treatment of severe retinopathy of prematurity (ROP), but there are no human data addressing potential systemic absorption and effects on systemic Veg F levels in preterm infants. We measured Veg F in preterm infants during their stay in intensive care including some infants treated with laser or bevacizumab for ROP.

Methods Serum and plasma were salvaged as part of a consented research study in preterm infants from delivery to discharge. Veg F levels were measured using flow cytometry and analysed in relation to gestation, postmenstrual age and any ROP treatment.

Results 26 infants, median 26 wks gestational age (range 23-29 wks) contributed 114 samples. 5 received laser treatment for ROP, 2 bevacizumab. Serum VegF levels decreased with increasing postmenstrual age. Levels for those treated for ROP did not differ from those untreated. The two infants that received bevacizumab had lower VegF levels after treatment than before.

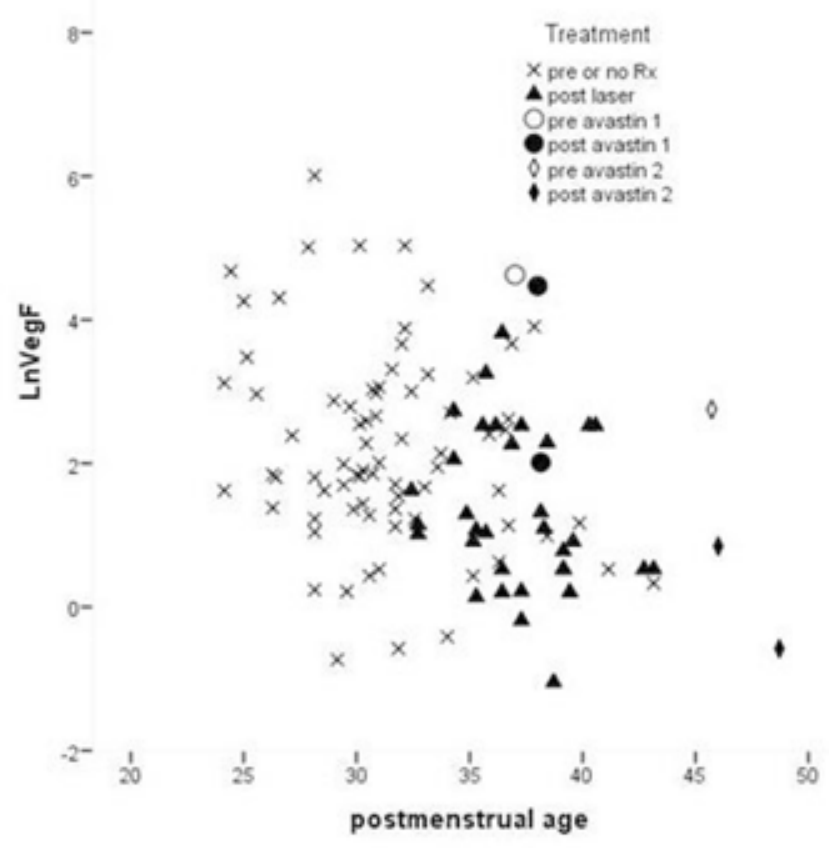

\section{Abstract 168 Figure 1}

Conclusions This first data in human preterm infants with and without ROP treatment show trends with increasing postmenstrual age, and that VegF levels in infants treated with bevacizumab do not appear to differ from those untreated or those treated with laser. Individual infants receiving bevacizumab had lower VegF levels in the first week after injection than before. As bevacizumab treatment increases efforts should be made to further assess effects on circulating VegF.

\section{HOSPITAL MORTALITY IN 2,437 INFANTS IN THE AUSTRALIAN, NEW ZEALAND AND UK BOOST II TRIALS OF NEONATAL OXYGEN SATURATION TARGETING}

doi:10.1136/archdischild-2012-302724.0169

1,2WO Tarnow-Mordi, ${ }^{3} \mathrm{~B}$ Stenson, ${ }^{4} \mathrm{~B}$ Darlow, ${ }^{5} \mathrm{P}$ Brocklehurst, ${ }^{6} \mathrm{C}$ Morley, ${ }^{6} \mathrm{P}$ Davis, ${ }^{5}$ E Juszczak, ${ }^{5} \mathrm{~A}$ King, ${ }^{7} \mathrm{~K}$ Simmer, ${ }^{2} \mathrm{~A}$ Kirby, ${ }^{2} \mathrm{M}$ Donoghue, ${ }^{2} \mathrm{~A}$ Ghadge, ${ }^{2} \mathrm{~W}$ Hague, For the Australian, New Zealand and UK BOOST II Collaborative Study Groups. 'WINNER Centre, NHMRC Clinical Trials Centre, University of Sydney; ${ }^{2}$ NHMRC Clinical Trials Centre, University of Sydney, Sydney, NSW, Australia; ${ }^{3}$ Simpson Centre for Reproductive Health, Royal Infirmary, Edinburgh, UK; ${ }^{4}$ Christchurch Women's Hospital, University of Otago, Christchurch, New Zealand; ${ }^{5}$ National Perinatal Epidemiology Unit (NPEU), University of Oxford, Oxford, UK; ${ }^{6}$ Royal Womens Hospital, University of Melbourne, Melbourne, VIC; ${ }^{7}$ King Edward Memorial Hospital, University of Western Australia, Perth, WA, Australia

Background The optimal oxygen saturation $\left(\mathrm{SpO}_{2}\right)$ for preterm infants is unknown. Three BOOST II trials in Australia, New Zealand and UK are comparing outcomes in infants $<28$ weeks after randomisation to $\mathrm{SpO}_{2}$ targeting of $85-89 \%$ vs $91-95 \%$, using masked oximeters. ${ }^{1}$ In interim analysis the high target increased 36 week survival in infants whose oximeter had been upgraded with new, more accurate software..$^{2,3}$

Methods Pooled analysis of hospital mortality by target, overall and by old or new software. 